\title{
Analysis of a Free Surface Film from a Controlled Liquid Impinging Jet over a Rotating Disk Including Conjugate Effects, with and without Evaporation
}

\author{
Jeremy Rice Amir Faghri Baki M. Cetegen \\ Department of Mechanical Engineering, University of Connecticut, Storrs, CT 06269-3139
}

\begin{abstract}
A detailed analysis of the liquid film characteristics and the accompanying heat transfer of a free surface controlled liquid impinging jet onto a rotating disk are presented. The computations were run on a two-dimensional axi-symmetric Eulerian mesh while the free surface was calculated with the volume of fluid method. Flow rates between 3 and $15 \mathrm{lpm}$ with rotational speeds between 50 and $200 \mathrm{rpm}$ are analyzed. The effects of inlet temperature on the film thickness and heat transfer are characterized as well as evaporative effects. The conjugate heating effect is modeled, and was found to effect the heat transfer results the most at both the inner and outer edges of the heated surface. The heat transfer was enhanced with both increasing flow rate and increasing rotational speeds. When evaporative effects were modeled, the evaporation was found to increase the heat transfer at the lower flow rates the most because of a fully developed thermal field that was achieved. The evaporative effects did not significantly enhance the heat transfer at the higher flow rates.

1 Introduction

Impinging jets on rotating surfaces have received a lot of attention lately because of the high potential of heat transfer rates that can be achieved. There are two major classes of impinging jets useful for engineering applications; they are the submerged jet and the free jet. A submerged jet is one that flows into a stagnant fluid of similar properties, whereas a free jet is a liquid that flows from a nozzle into a gaseous region, and is virtually unaffected by gas, hence the term free. There are numerous applications of impinging jets, which include material processing, turbine blade cooling and cooling of electronics. In the present study, a controlled liquid impinging jet is studied. A controlled liquid impinging jet flows onto a planar disk; near the center of the disk there is another smaller coaxial disk (collar), which controls the entrance free film thickness and entrance radius of the free film. The present study is motivated because of the envisioned potential of rotating thin film fluids in an absorber unit for a spacecraft vapor-absorption heat pump system. This unit will work in a micro-gravity environment, where the disk can be rotated to apply an additional force to thin the fluid and enhance the heat transfer.
\end{abstract}

Watson [1] first studied a free liquid jet impinging normally to a planar surface. He assumed that a substantial portion of the flow near the stagnation point was inviscid and was not affected by the planar surface. He used a similarity solution with a boundary layer approximation to determine the film thickness. Chaudhury [2] studied the heat transfer to Watson's analysis in a similar manner. Craik et al. [3] studied the hydraulic jump formed by an impinging jet of liquid onto a planar surface. They took film thickness measurements with a light absorption technique and also did flow visualization. They found a presence of an eddy just downstream of the hydraulic jump. Tong [4] numerically studied a planar liquid jet impinging obliquely to a planar surface. He used the volume of fluid method to track the free surface. The study focused on heat transfer near the stagnation region, and where the maximum heat transfer occurs.

Rahman et al. [5] developed a finite difference solution using boundary-fitted curvilinear coordinates to locate the free surface. In this technique the free surface conformed to one of the computational boundaries. They studied a falling film down a vertical wall; they also studied plane and radial film flows with and without gravity. Faghri et al. [6] later used this numerical technique to model heat transfer from a controlled impinging jet onto a stationary disk with the conjugate heating effects included. The numerical data were compared to the experimental data, and if the conjugate heating effects were not included, an error as large as 100 percent was seen. Thomas et al. [7] experimentally studied the film thickness of a controlled impinging jet on a stationary and rotating disk. The film thickness measurements were taken using a capacitance technique. The research found that the film thickness on the rotating disk was affected by the inertial and frictional forces on the fluid near the center of the disk, and centrifugal forces near the outer edge of the disk. Also the hydraulic jump was found to "wash off" the disk in the presence of a rotational field. Thomas et al. [8] performed a one-dimensional analysis of the hydrodynamic and thermal characteristics of thin film flows with and without rotation with the MacCormack predictor-corrector method. The hydraulic jump was not present in the absence of gravitational forces. However, when the disk was rotated the film thickness was 
unaffected by gravitational forces. The heat transfer was found to be much greater with rotation because the liquid film is much thinner and is moving with a higher velocity.

Rahman and Faghri [9] solved a three dimensional slice of the flow over a rotating disk using boundary-fitted coordinates; also they improved on Thomas et al.'s [8] model by better approximating the frictional resistance in the azimuthal direction exerted by the solid wall on the flow. They ran cases with heating as well as with evaporation and found that increased flow rate and rotational speed both increased the rate of heat transfer. Most recently, Ozar et al. [10] experimentally studied the heat transfer effects of a thin liquid film from a controlled impinging jet on both a stationary and rotating disk. The fluid mechanics and the heat transfer characteristics were examined simultaneously. Semi-empirical correlations were found to characterize both the local Nusselt number and the average Nusselt number.

From the above literature review, previous numerical models solving liquid jet impingement on a rotating disk mainly involve trial and error methods, which require guessing at the free surface location in some systematic incremental sequence. In the present study, the volume of fluid method is used to track the free surface. This technique requires a single domain, time-dependent solution, which directly locates the free surface once a steady-state solution is achieved, resulting in a highly accurate computation. Furthermore, the free surface has surface tension effects included, which have not previously been modeled properly. The present analysis also includes a conjugate heating effect in the disk, which is sparse in the previous computational work as a single domain problem, and helps to draw better correlations with existing experimental data. Effects of inlet temperatures and flow conditions are studied, which affect both the flow and thermal characteristics of the system. The present analysis also include evaporative cases, or purely heating cases when evaporation is considered negligible. Whether evaporation is important or not is determined by the inlet temperature of the fluid.

\section{Nomenclature}

A: $\quad$ Heater area $\pi\left(\mathrm{r}_{\text {hout }}{ }^{2}-\mathrm{r}_{\text {hin }}{ }^{2}\right)\left(\mathrm{m}^{2}\right)$

c: Discharge coefficient for impinging jets

C: $\quad$ Specific heat (joules $/ \mathrm{kg}-\mathrm{K}$ )

$\mathrm{d}_{\mathrm{d}}$ : $\quad$ Disk thickness $(\mathrm{m})$

F: $\quad$ Body force due to surface tension $\left(\mathrm{N} / \mathrm{m}^{3}\right)$

g: Gravity $\left(\mathrm{m} / \mathrm{s}^{2}\right)$

h: $\quad$ Enthalpy (joules $/ \mathrm{kg}$ )

$\mathrm{k}$ : Thermal conductivity (W/m-k)

$\dot{m}$ : $\quad$ Mass flow rate $(\mathrm{kg} / \mathrm{s})$

$\vec{n}: \quad$ Normal unit vector

$\mathrm{Nu}$ : Local Nusselt number at the disk/liquid interface

$$
\left(\frac{q^{n} r_{i n}}{\Delta T \cdot k_{l}}\right)
$$

$\overline{N u}: \quad$ Average Nusselt number $\left(\frac{1}{A} \int_{A} N u d A\right)$

p: $\quad$ Pressure $(\mathrm{Pa})$

$\dot{q}^{n}: \quad$ Heat flux, Q/A, $\left(\mathrm{W} / \mathrm{m}^{2}\right)$
Q: $\quad$ Heater power, (W)

r: $\quad$ Radial coordinate $(\mathrm{m})$

$r_{t}: \quad$ Laminar-turbulent transition radius $(m)$

$r_{t}^{*}$ : Dimensionless laminar-turbulent transition radius

$\frac{r_{t}}{\mathrm{Re}^{* 1 / 3} \sqrt{c 2 r_{\text {hin }} \delta_{\text {in }}}}$

Re: $\quad$ Reynolds number $\left(\frac{\dot{m}}{\mu 2 \pi r_{h i n}}\right)$

Re*: Reynolds number used by Azuma and Hoshino (1984)

$\left(\frac{\dot{m}}{\mu \sqrt{c 2 r_{\text {hin }} \delta_{\text {in }}}}\right)$

t: $\quad$ Time (s)

$\bar{t}: \quad$ Tangential unit vector

T: $\quad$ Temperature $(\mathrm{K})$

$\Delta \mathrm{T}: \quad$ Temperature difference, $\mathrm{T}_{\mathrm{w}}-\mathrm{T}_{\text {in }}(\mathrm{K})$

u: $\quad$ Axial velocity $(\mathrm{m} / \mathrm{s})$

v: Radial velocity $(\mathrm{m} / \mathrm{s})$

$\vec{V}: \quad$ Velocity vector $(\mathrm{m} / \mathrm{s})$

$\dot{V}: \quad$ Volumetric flow rate (lpm)

w: $\quad$ Swirl velocity $(\mathrm{m} / \mathrm{s})$

$\mathrm{x}$ : Axial coordinate (m)

Greek Symbols:

$\alpha$ : Volume fraction of liquid

$\delta: \quad$ Film thickness (m)

$\phi$ : $\quad$ General volume weighted fluid property

$\kappa:$ Total curvature $\left(\mathrm{m}^{-1}\right)$

$\mu: \quad$ Dynamic viscosity $(\mathrm{kg} / \mathrm{m}-\mathrm{s})$

$\rho: \quad$ Density $\left(\mathrm{kg} / \mathrm{m}^{3}\right)$

$\sigma: \quad$ Surface tension $(\mathrm{N} / \mathrm{m})$

$\omega: \quad$ Angular velocity $(\mathrm{rad} / \mathrm{s})$

\section{Subscripts:}

hin: Inner radius of heater

hout: Outer radius of heater

i: Counting index

in: Inlet

1: Liquid

ref: Reference

t: $\quad$ Laminar-turbulent transition

v: $\quad$ Vapor

w: Disk surface

\section{Analysis}

Figure 1 shows a schematic of the system being modeled. Flow enters the disk between two circular plates, one being the collar, and the other being the disk. The spacing between the collar and the disk is $\delta_{\text {in }}$. After the flow leaves the entrance region between the collar and the disk at $r_{\text {hin }}$, the flow turns from an internal fully developed flow to a free surface flow. The heater provides a constant heat flux at this point, from the bottom of the aluminum disk. The heat is conducted 


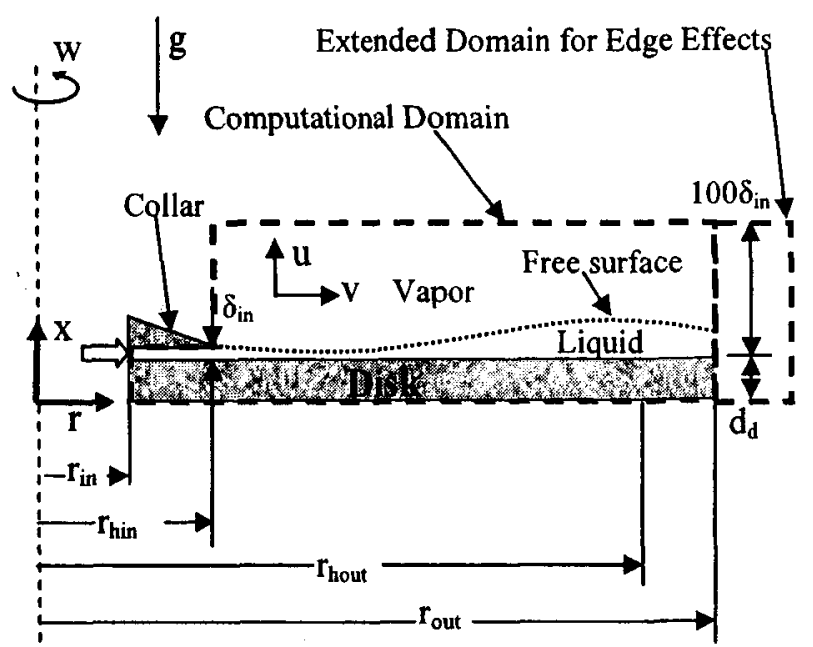

through the disk to the fluid. The basic assumptions of the problem at hand are that the flow field is incompressible, the fluid is considered to be in the laminar flow regime while all of the fluid properties are constant. When evaporation is being modeled, no mass transfer is modeled and the effects are only considered in the energy equation, because the evaporation rate of the liquid is much less than the inlet mass flow rate of the liquid. The Navier-Stokes equations are solved to compute the fluid flow. The continuity, and momentum equations are as follows:

$$
\begin{gathered}
\nabla \cdot \vec{V}=0 \\
\rho \frac{D \vec{V}}{D t}=-\nabla p+\nabla \cdot(\mu \nabla \vec{V})+\rho g+F
\end{gathered}
$$

Since the flow field is assumed to be independent of the temperature field, a steady-state energy equation is solved in the fluid region, once the flow field has been resolved.

$$
\nabla \cdot(\bar{V} \rho h)=\nabla \cdot(k \nabla T)
$$

In the solid region, only conduction is solved.

$$
\nabla^{2} T=0
$$

The free surface is tracked by the Volume of Fluid (VOF) method developed by Hirt and Nichols [11], where the volume fraction, $\alpha$, of the fluid is tracked through each computational cell. The VOF equation is:

$$
\frac{\partial \alpha}{\partial t}+\vec{V} \cdot \nabla \alpha=0
$$

The interface between fluids is represented by a piecewise linear approach, similar to the work of Youngs [12], to greatly limit numerical diffusion of the interface. Surface tension effects are modeled in the present numerical simulations. The surface tension forces are represented by the " $F$ " term in
Equation 2. A continuum surface force method proposed by Brackbill [13] is used to model surface tension.

$$
F=\sigma \frac{\rho \kappa \nabla \alpha}{0.5\left(\rho_{l}+\rho_{v}\right)}
$$

The curvature, $\kappa$, is defined as:

$$
\kappa=\frac{\nabla^{2} \alpha}{|\nabla \alpha|}
$$

The fluid properties are calculated by the volume weighted average.

$$
\phi=\alpha \phi_{1}+(1-\alpha) \phi_{v}
$$

The general fluid property, $\phi$, represents density, viscosity and thermal conductivity. The enthalpy is calculated using a massweighted average, instead of a volume-weighted average.

$$
h=\frac{\left[\alpha \rho_{l} C_{1}+(1-\alpha) \rho_{v} C_{v}\right]\left(T-T_{r e f}\right)}{\rho}
$$

The boundary conditions are as follows:

At the inlet $\left(r=r_{\text {ln }}\right)$

$$
\begin{aligned}
& \left(0<x<\delta_{i n}\right): v=v_{i n}, T=T_{i n} \\
& \left(-d_{d}<x<0\right): \frac{\partial T}{\partial r}=0
\end{aligned}
$$

At the collar $\left(x=\delta_{i n}, r_{i n}<r<r_{\text {hin }}\right)$

$$
u=v=0, w=\omega r, \frac{\partial T}{\partial x}=0
$$

At the disk surface $\left(x=0, r_{\text {in }}<r<r_{\text {out }}\right)$

$$
u=v=0, w=\omega r,\left.k \frac{\partial T}{\partial x}\right|_{,}=\left.k \frac{\partial T}{\partial x}\right|_{s}
$$

At the disk bottom $\left(x=-d_{d}\right)$

$$
\begin{array}{ll}
\left(r_{\text {in }}<r<r_{\text {hin }}\right): & \left.k \frac{\partial T}{\partial x}\right|_{s}=0 \\
\left(r_{\text {hin }}<r<r_{\text {hout }}\right): & \left.k \frac{\partial T}{\partial x}\right|_{s}=q^{\prime \prime}
\end{array}
$$




$$
\left(r_{\text {hot }}<r<r_{\text {out }}\right):\left.\quad k \frac{\partial T}{\partial x}\right|_{s}=0
$$

The above variation in heat flux is used to simulate the experimental prediction of Ozar et al. [10].

\section{At the outer boundaries}

$$
\begin{aligned}
& \left(r=r_{\text {hin }}, \delta_{\text {in }}<x<100 \delta_{\text {in }}\right),\left(r_{\text {hin }}<r<r_{\text {out }}, x=100 \delta_{\text {in }}\right) \\
& \left(r=r_{\text {out }}, \delta_{\text {in }}<x<100 \delta_{\text {in }}\right) \\
& \qquad \begin{array}{c}
p=p_{\text {ref }} \\
\text { if }(\vec{V} \cdot \vec{n}>0) \frac{\partial T}{\partial \vec{n}}=0 \text { else } T=T_{\text {ref }}
\end{array}
\end{aligned}
$$

At the liquid-vapor interface

$$
\begin{gathered}
p_{l}-p_{v}-\left.\mu_{;} \frac{\partial \vec{V} \cdot \vec{n}}{\partial \vec{n}}\right|_{l}+\left.\mu_{v} \frac{\partial \vec{V} \cdot \vec{n}}{\partial \vec{n}}\right|_{v}=\sigma \kappa \\
\left.\mu_{l} \frac{\partial \vec{V} \cdot \vec{t}}{\partial \vec{n}}\right|_{l}=\left.\mu_{v} \frac{\partial \vec{V} \cdot \vec{t}}{\partial \vec{n}}\right|_{v} \\
\text { heating: }\left.k_{l} \frac{\partial T}{\partial \vec{n}}\right|_{l}=\left.k_{v} \frac{\partial T}{\partial \vec{n}}\right|_{v}, \\
\text { evaporation: } T=T_{\text {sat }}
\end{gathered}
$$

Air was used as the vapor for all of the simulations. The viscosity and thermal conductivity of air were lessened by an order of magnitude for selective cases, and were found not to change the results; therefore, the boundary condition at the interface is essentially a zero shear stress for all cases, and an insulated boundary for the heating cases.

As was noted before, the flow field is considered to be independent of the thermal field; therefore the flow field is first solved as a time-dependent solution, until a steady state solution has been reached. Once a steady-state solution has been reached, the energy equation is solved for both the fluid and solid regions, as a steady-state solution. The criteria used to determine a steady-state solution was when the mass flow rate out of the domain was within $0.05 \%$ of the mass flow rate into the domain for 0.05 seconds. The flow field was solved using the following procedure:

1. Solve momentum equations

2. Solve continuity (pressure correction) equation, update pressure and face mass flow rate

3. Repeat steps 1 and 2 until converged, for each time step

A co-located finite volume computational scheme, where both the flow-field variables and the pressure are stored in the cell centers, is used to solve the governing equations. The pressure is discretized in a manner similar to a staggered-grid scheme, while the pressure and velocity are coupled using the SIMPLE algorithm, described by Patankar [14]. All of the convective terms in the governing equations are discretized using a second-order upwind scheme.
The grid used for the numerical simulations consisted of rectangular shaped cells, which were produced in four axial layers in the liquid region, and one axial layer in the solid region. In the fluid region in the first layer, spanning from the disk surface to $\delta_{\text {in }}$, there were 25 cell rows, with a growth rate of 1.01. The second layer, spanning from $\delta_{\text {in }}$ to $3 \delta_{\text {in }}$, consisted of 30 cell rows with a growth rate of 1.02 . The third layer, spanning from $3 \delta_{\text {in }}$ to $10 \delta_{\text {in, }}$ consisted of 25 cell rows, and had a growth rate of 1.05 . In the final layer, spanning from $10 \delta_{\text {in }}$ to $100 \delta_{\text {in }}$, there were 20 cell rows, with a growth rate of 1.2 . In the solid region, there were 15 evenly spaced cell rows. In the radial direction, there were 15 evenly spaced cell columns between $r_{\text {in }}$ and $r_{\text {hin }}$, and 75 evenly spaced cell columns between $r_{\text {hin }}$ and $r_{\text {out }}$. The grid spans such a great distance in the axial direction $\left(100 \delta_{\text {in }}\right)$, because convergence is greatly increased with the added cells, so the computation actually runs more rapidly. A grid study was performed on a mesh that had 35 cell rows growing at a rate of 1.01 in the first layer of the liquid. The second layer had 40 cell rows with an aspect ratio of 1.02 . The third layer had 25 cell rows with an aspect ratio of 1.075 , and the fourth layer had 20 cells with an aspect ratio of 1.2. In the solid region there were 25 evenly spaced cell rows. In the radial direction, there were 15 cell columns between $r_{\text {in }}$ and $r_{\text {hin, }}$ and 100 evenly spaced cell columns between $r_{\text {hin }}$ and $r_{\text {out }}$. The film thicknesses as well as the Nusselt numbers for both computational grids were within 0.1 percent of each other for all radial locations, for the $7 \mathrm{lpm}, 100 \mathrm{rpm}, 40^{\circ} \mathrm{C}$ case.

\section{Results and Discussion}

The disk has the same dimensions for all of the cases to simulate the experimental data of Ozar et al. (2004). The inner radius of the disk, $r_{\text {in }}$, is $0.0254 \mathrm{~m}$. The inner radius of the heater, and the exit of the collar, $r_{\text {hin }}$, is $0.0508 \mathrm{~m}$, while the outer radius of the heater, $r_{\text {hout }}$ is $0.18 \mathrm{~m}$. The outer radius of the disk, $r_{\text {out }}$, is $0.2032 \mathrm{~m}$. The aluminum disk thickness is $0.00635 \mathrm{~m}$ unless otherwise noted. The working fluid for all of the simulations is water. The thermal conductivity of aluminum is $202.4 \mathrm{~W} / \mathrm{m}-\mathrm{K}$ for all of the cases. This set-up is used to examine film thickness and heat transfer measurements for flow rates ranging from 3 to $15 \mathrm{lpm}$, and disk rotational speeds of 50 to $200 \mathrm{rpm}$. The effects of inlet temperature are considered by varying the inlet temperature between $20^{\circ} \mathrm{C}$ and $100^{\circ} \mathrm{C}$. The effects of purely heating and evaporation are also presented. The purely heating boundary condition is used for the cases with inlet temperatures of $20^{\circ} \mathrm{C}$ and $40^{\circ} \mathrm{C}$, and the evaporation boundary condition is used for cases with an inlet temperature of $100^{\circ} \mathrm{C}$.

\subsection{Film Dynamics}

A prelude to the heat transfer characteristics of a controlled liquid impinging jet on a rotating surface is the fluid mechanics. In order to validate the present numerical schemes, the film thickness measurements are compared with experimental data obtained by two different techniques. The first technique, used by Thomas et al. [7], used a capacitance sensor. The present numerical results and the experimental results using the capacitance technique for the cases that matched the previously published data are presented in Figure 2. Since there were waves present on the surface of the liquid film in the experimental study, data were sampled at a rate of 


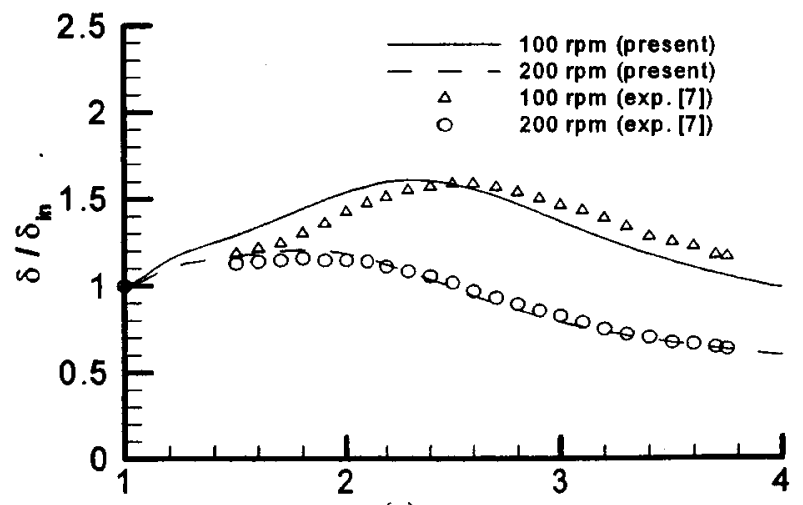

(a)

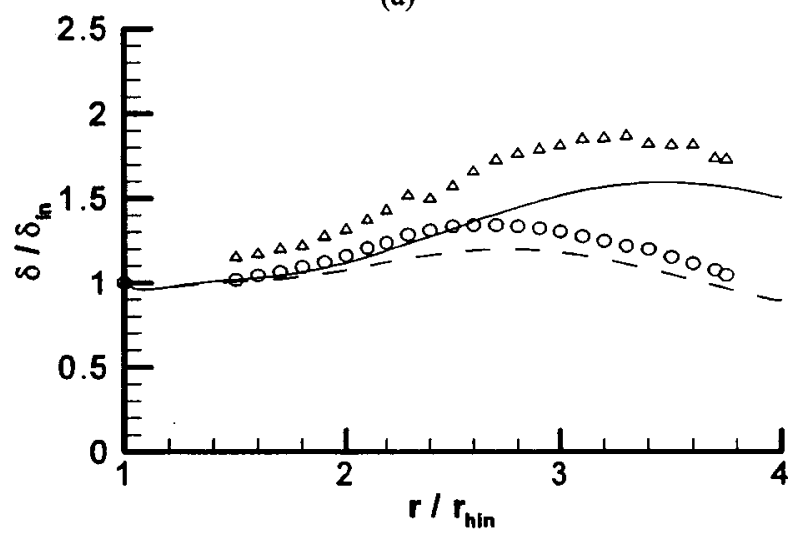

(b)

Flgure 2: Comparison of Numerical and Experimental results of the film thickness vs. radial distance at an inlet temp of $20^{\circ} \mathrm{C}, \delta_{\text {in }}=0.254 \mathrm{~mm}$ (Num.) and $0.267 \mathrm{~mm}$ (exp.), and flow rates of (a) $7 \mathrm{lpm}$ and (b) $15 \mathrm{lpm}$

one every two seconds, for every radial location, to obtain a repeatable average measurement. The maximum difference between the numerical and the experimental data for the $7 \mathrm{lpm}$, 100 and $200 \mathrm{rpm}$ cases, and the $15 \mathrm{lpm}, 100$ and $200 \mathrm{rpm}$ cases are 8.8, 3.9, 22.0 and 13.3 percent, respectively.

The present numerical film thickness measurements are also compared with experimental data obtained by Ozar et al. [15], in Figure 3. These experimental data were gathered by a laser light reflection technique, and images of the reflections of the dry and wet disk surfaces were digitally combined to obtain an image, which was post-processed to extract the center points of the reflections to determine the liquid film thickness. The slight wobbling of the disk was accounted for by triggering the camera at the same angular location. The final data points were fit with a least squares fourth-order polynomial. There is no mention of the repeatability of the published data, or variation with angular position. The numerical prediction tends to capture a thicker film thickness than the experimental predictions for the 3 and $7 \mathrm{lpm}$ cases, for all of the presented rotational speeds. The opposite effect was observed for the 15 lpm cases. The differences may arise from modeling the flow as an axisymmetric geometry, which cannot capture surface waves.

The effects of flow rate, rotational speed and inlet temperature are displayed in Figure 4. The physical parameters that affect the flow are the inlet inertia of the impinging jet and the viscous interaction with the fluid and the disk. The viscous

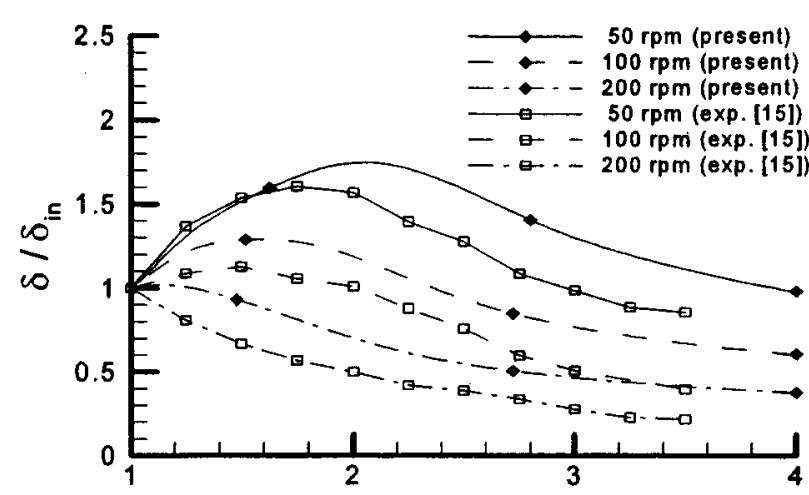

(a)

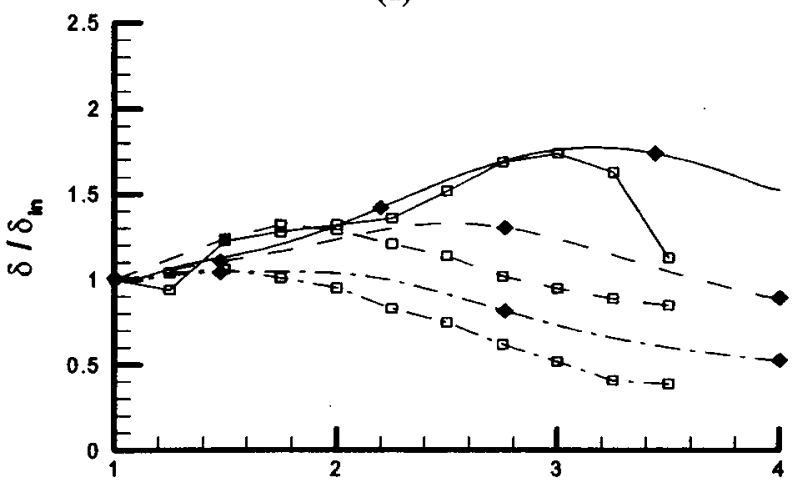

(b)

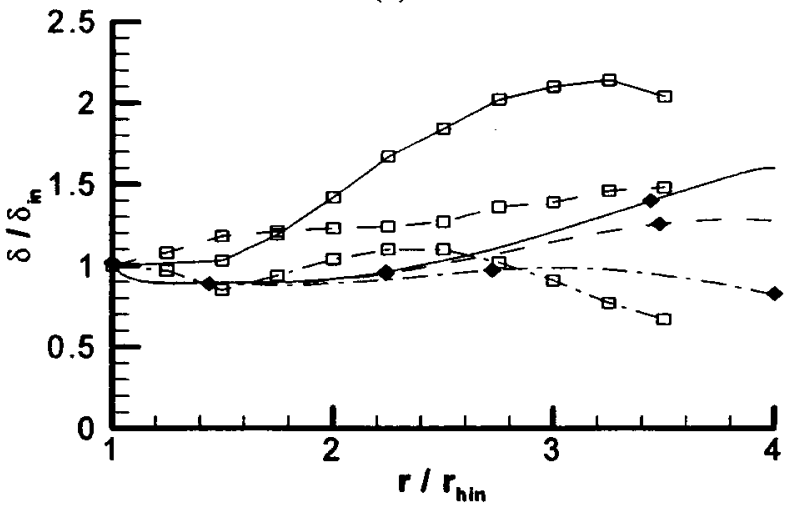

(c)

Figure 3: Comparison of Numerical and Experimental film thickness measurements vs. radial distance at $40^{\circ} \mathrm{C}$ inlet temperature, $\delta_{\text {in }}=0.254 \mathrm{~mm}$ at flow rates of (a) $3 \mathrm{lpm}$ (b) $7 \mathrm{lpm}$ (c) $15 \mathrm{lpm}$

interaction between the disk and the liquid both retards the flow in the radial direction, and adds a centrifugal force because of the rotation of the disk. The film thickness generally increases due to the frictional losses of the fluid, and then decreases due to the centrifugal forces. The film thickness decreases with increasing rotational speed. Also the point where the maximum film thickness is located moves closer to the collar with increasing rotational speed. These two observed behaviors occur because the centrifugal force has an increased magnitude with increased rotational speed. The point of maximum film thickness moves closer to the outer edge of the disk as the flow rate is increased because the inertia of the fluid is greater, therefore taking a greater distance for the frictional forces of 


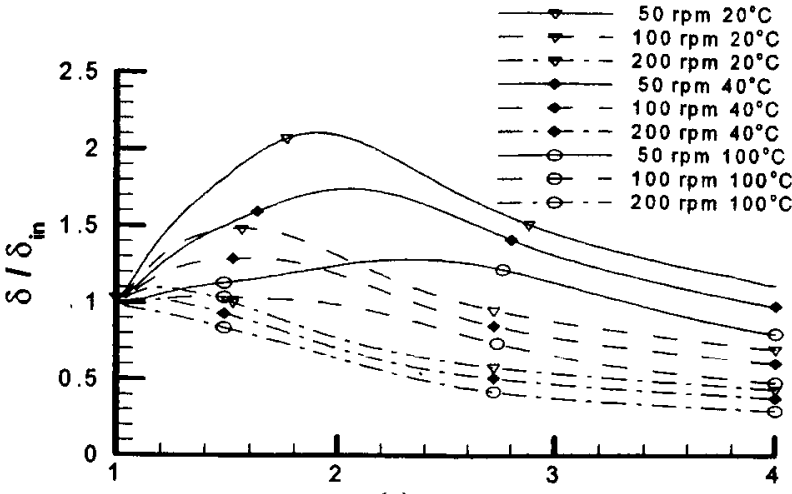

(a)

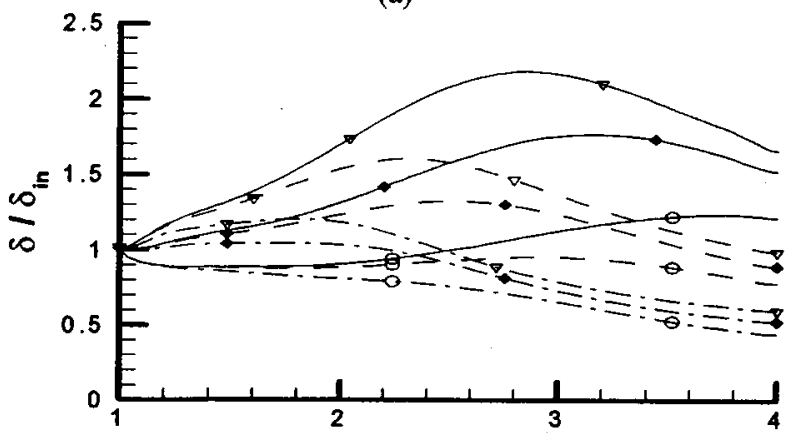

(b)

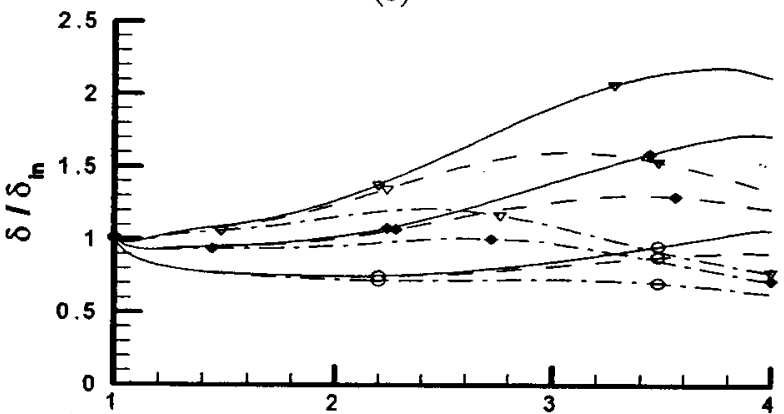

(c)

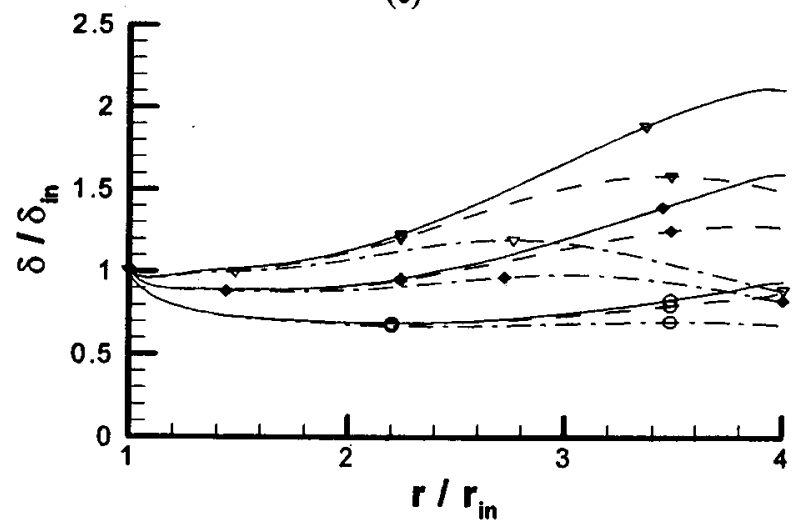

(d)

Figure 4: Numerical Film Thickness data vs. radial distance with $\delta_{\text {in }}=0.254$ $\mathrm{mm}$ at various temperatures and flow rates of (a) $3 \mathrm{lpm}$ (b) $7 \mathrm{lpm}$ (c) $12 \mathrm{lpm}$ (d) $15 \mathrm{lpm}$

the disk to retard the flow. A better indicator of when the centrifugal forces begin to influence the film thickness is to look at where the film thickness heights for the same flow rate

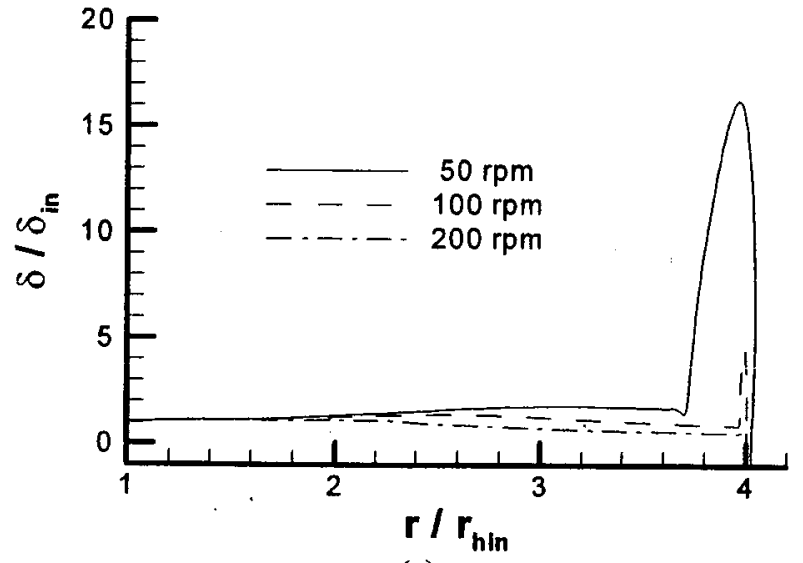

(a)
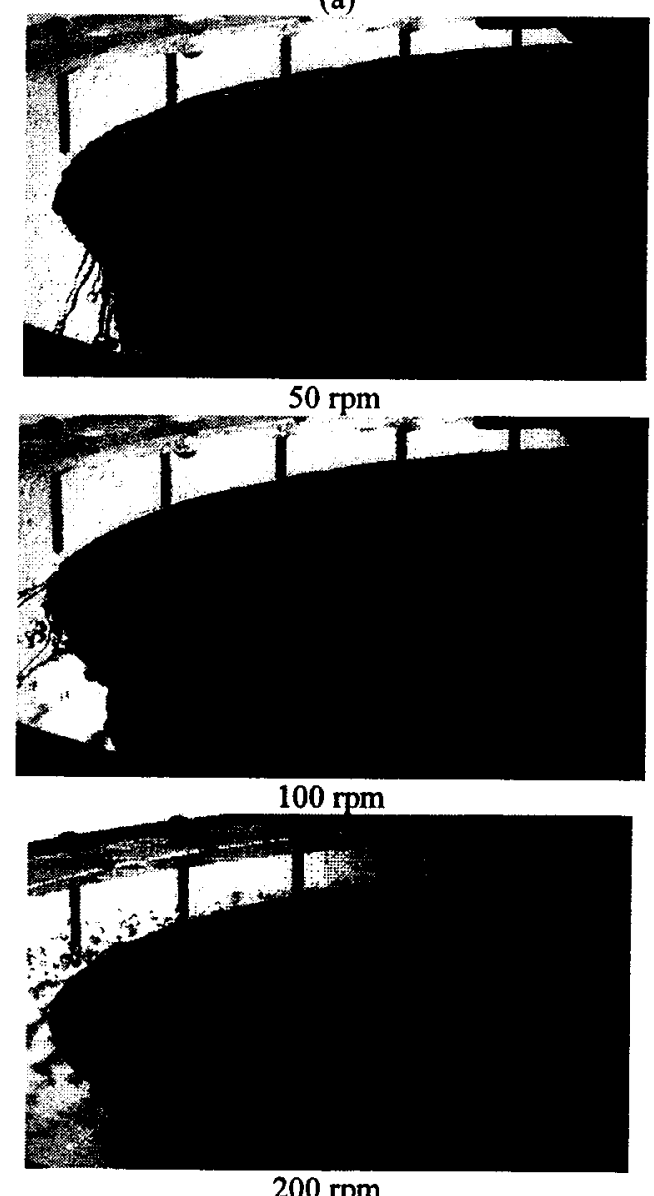

(b)

Figure 5: (a) Numerical Film Thickness data vs. radial distance including edge effects and (b) experimental flow visualization with $\delta_{\mathrm{in}}=0.254 \mathrm{~mm}, 7 \mathrm{lpm}$ and $40^{\circ} \mathrm{C}$ inlet temperature [15]

begin to vary with rotational speed. For the lower flow rates, rates there is a large amount of overlapping of the film thicknesses over the disk surface, and the distinction between the different rotational speeds is not realized until further out on the disk. The effects of increasing temperature tend to reduce the film thickness because the frictional losses are less due to the decrease in viscosity. Also, the point where the maximum film thickness occurs moves radially outward with increasing 
temperature because the rotational effects from the rotation of the disk do not diffuse as readily to the bulk fluid movement with the decrease in viscosity.

Figure 5 illustrates the free surface shape when the edge effects are included in this analysis. The edge effects come from the fluid flowing over the edge of the disk, therefore causing a surface curvature. This surface curvature, raises the pressure because of the surface tension, and causes the fluid to make a lip at the disk's edge. This lip is commonly referred to as a "hydraulic jump". The film thickness of the model with the edge effects is within 2 percent of the model without the edge effects being modeled before a value of $r / r_{\text {hin }}$ of 3.5 . There is a hydraulic jump at the edge of the disk that decreases in magnitude as the rotational speed is increased. This result is qualitatively consistent with the edge effects observed by Ozar et al. [15]. The hydraulic jump at the edge of the disk is caused by the surface tension of the fluid. As the fluid flows over the edge, the surface curvature increases, therefore increasing the pressure. This increased pressure retards the flow and causes a jump. When rotational speeds are increased, the centrifugal forces increase, therefore making the edge effects have a relatively weaker resistance to the flow and washing off the hydraulic jump. It is important to note that there are surface waves present on the liquid film in the experiment that are not captured in the numerical model because of the axisymmetric assumption.

\subsection{Thermal Characteristics}

Now that an understanding of the liquid film flow characteristics is established, the heat transfer from the disk to the fluid can be investigated. Even though the conjugate effect is modeled, the Nusselt numbers are defined by the applied heat flux of the heater $(\mathrm{Q} / \mathrm{A})$, not the actual heat flux at the disk surface, and the temperature difference between the disk surface and the inlet temperature. This definition is used so that comparisons can be made with experimental data, in which only the heat flux at the heater is known. Figure 6 shows a comparison of local Nusselt numbers for various rotational speeds and flow rates of the present numerical simulation with the experimental data taken by Ozar et al. [10]. The numerical and experimental results correlate well at the larger radii, and increasingly poor at inner radii with increasing flow rate. The experimental Nusselt numbers at the inner radii are always lower than of the numerical results meaning that the surface temperatures at these locations are higher in the experiments. The differences may arise from non-uniform heating of the disk in the experiments because of the varying contact pressure of the heater. The contact pressure may vary because of the difference in coefficients of thermal expansion in the aluminum disk and the ceramic insulating layer.

The local Nusselt number versus radial location for various flow rates and rotational speeds are presented in Figure 7. The only variable in the definition of the local Nusselt number is the inverse of the temperature difference between the disk surface and the fluid inlet. The heater power for these cases is 4500 watts. The wall temperature starts off at the lowest value at the inlet of the impinging jet because the mean temperature of the fluid is the lowest at this point. As the mean temperature of the fluid increases as it flows and heats up over the disk, so does the disk's surface temperature. The conjugate

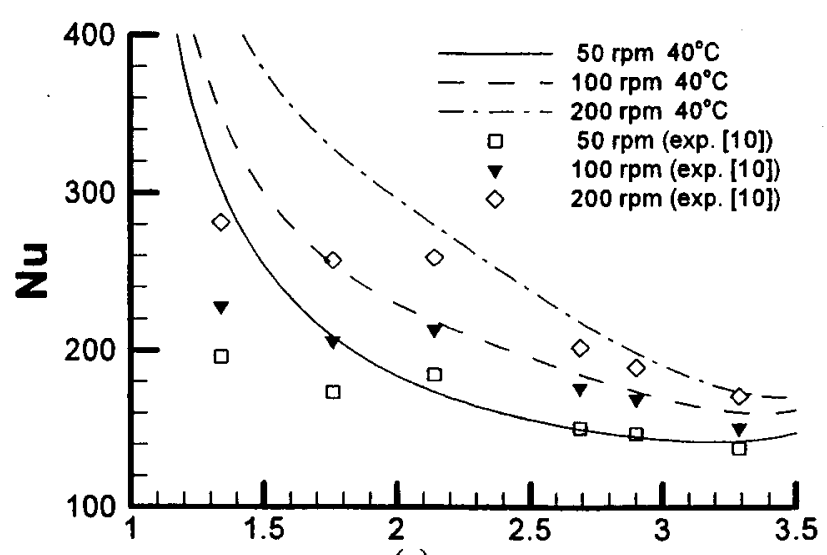

(a)

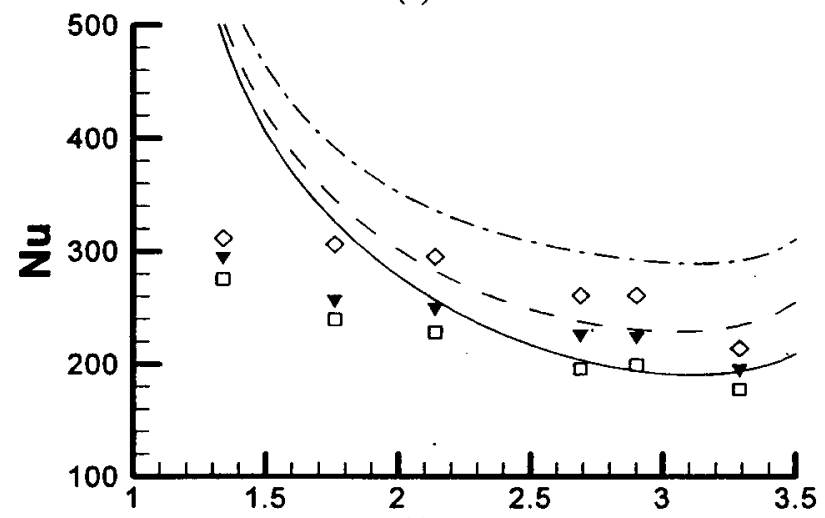

(b)

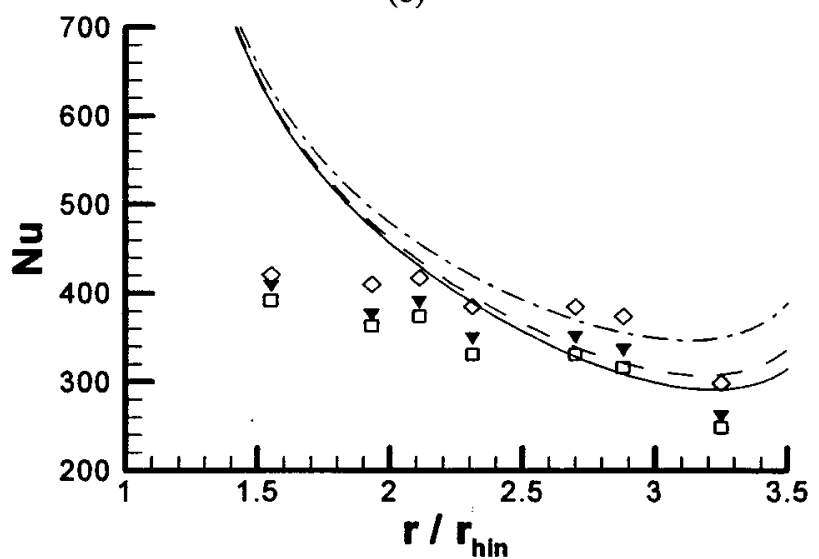

(c)

Figure 6: Comparison of Numerical and Experimental Local Nusselt Numbers vs. radial distance based on inlet temperature and radius with $\delta_{\text {in }}=0.254 \mathrm{~mm}$, and an inlet temperature of $40^{\circ} \mathrm{C}$ for flow rates of (a) $3 \mathrm{lpm}$ (b) $7 \mathrm{lpm}$ (c) 15 Ipm

heating effect is noticeable because the surface temperature actually decreases towards the end of the heater. The outer decrease in temperature occurs because of radial conduction within the disk. A more quantitative look at conjugate heating is discussed in the latter part of the thermal effects.

The surface temperature is also a function of the inlet flow rate, rotational speed, and inlet temperature. As the rotational speed increases, the surface temperature decreases, because the liquid film thickness decreases, which increases the 


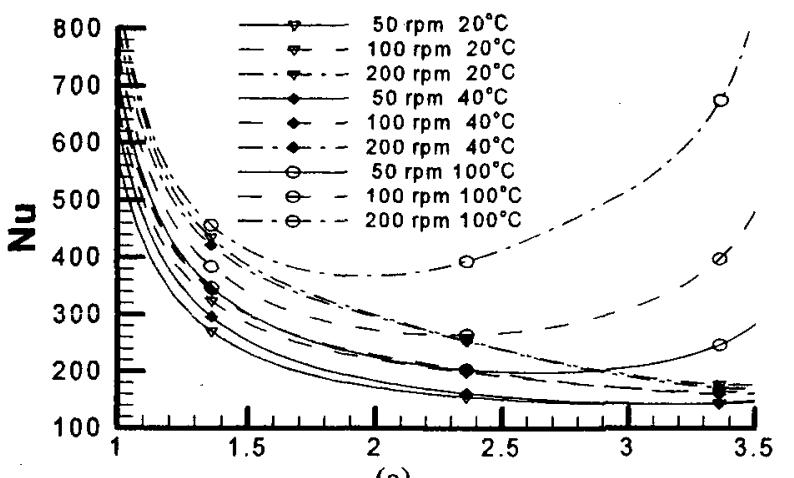

(a)

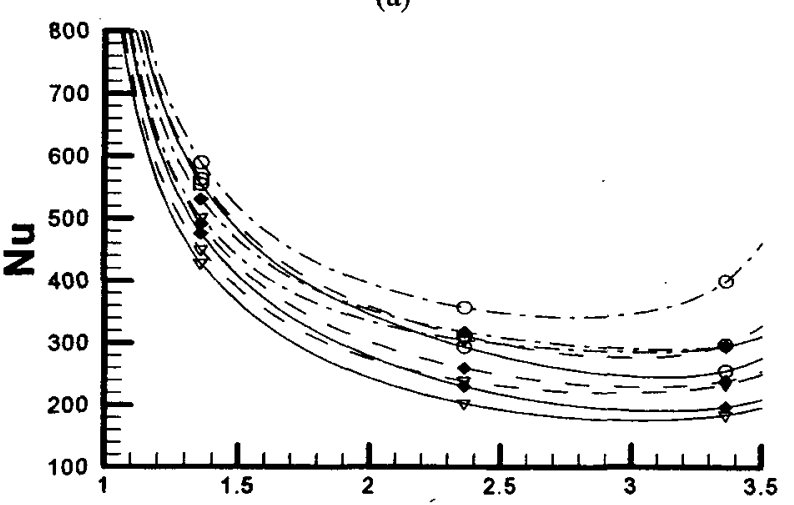

(b)

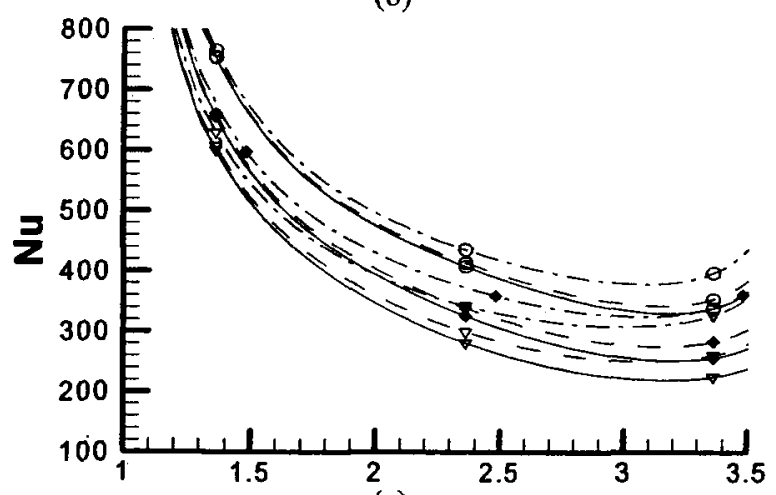

(c)

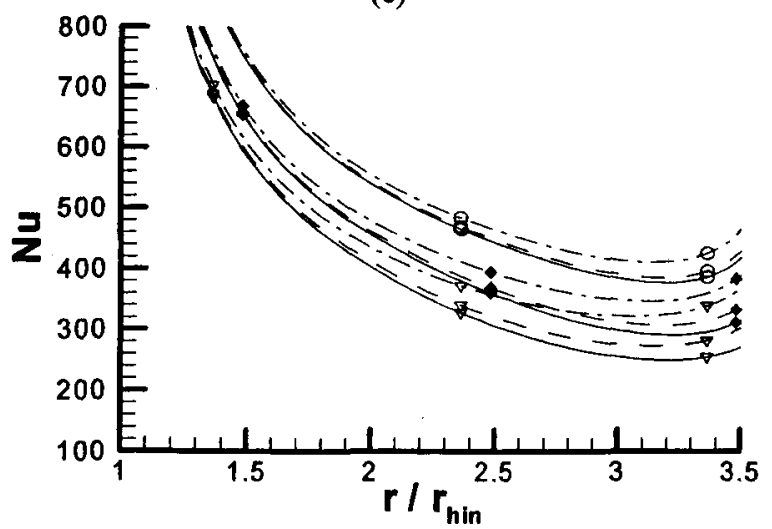

(d)

Figure 7: Local Nusselt Number vs. radial distance of liquid impinging jet on a rotating disk, $\delta_{\mathrm{in}}=0.254 \mathrm{~mm}$, and a heating power of 4500 watts (a) $3 \mathrm{lpm}$ (b) 7 $\mathrm{lpm}$ (c) $12 \mathrm{lpm}$ (d) $15 \mathrm{lpm}$ mean fluid velocity at each radial location. The surface temperature goes down with increasing flow rate, because the mean fluid temperature is lower. The increased inlet temperature decreases the viscosity, which thins the film. The fluid velocity is higher because of the thinner film, consequently decreasing the temperature difference between the disk and the inlet.

For the lower flow rates, the $20^{\circ} \mathrm{C}$ and $40^{\circ} \mathrm{C}$ inlet temperature cases show minimal difference in local Nusselt number, the differences are mainly seen with the increase in rotation. This result occurs because the fluid film characteristics are more strongly a function of rotational speed and than the liquid properties examined. At higher flow rates, the Nusselt Number distribution is affected more by temperature at the inner radii and more strongly by rotational speed at the outer radii. This trend physically occurs because the film characteristics vary more significantly with rotational speed at outer radii and vary more significantly with temperature at inner radii.

The effects of evaporation can be seen in the $100^{\circ} \mathrm{C}$ inlet temperature cases. The enhancement of heat transfer because of the evaporative effects are the most significant in the lower flow rate cases, and increase rapidly in these cases with rotational speed. This trend comes about because the thermal characteristics of the fluid resemble a fully developed thermal field at lesser radii for lower flow rates; therefore temperature profile throughout the film thickness is nearly linear. When the film thickness decreases, the surface temperature also decreases because temperature gradient throughout the liquid film remains constant. At higher flow rates, the flow is thermally developing throughout the heated region and has more of a parabolic temperature profile throughout the film thickness for most of the radii that are heated. Therefore, the evaporative cooling effects are less significant at higher flow rates. Heater powers of 2000 and 9000 watts were used to check the impact heater power had on the results. Even though the film temperature rose significantly, the Nusselt number remained within 0.5 percent for all of the evaporative cases that were run. The heater powers that are presently used are low enough that no nucleate boiling is expected to occur. The purely evaporative mode of mass transfer is observed in rotational cases under similar heating loads in experiments that are currently being run to observe the effects of boiling/evaporation.

The effects of inlet temperature, flow rate and rotational speed have been characterized for their affects on local Nusselt numbers. Now the overall heat transfer performance of the disk used in these numerical simulations are displayed by means of average Nusselt number versus flow rate in Figure 8. The data points are fit with a fourth order polynomial. It is very clear the overall heat transfer is increased with flow rate and with rotational speed. The overall heat transfer changes very little in the $20^{\circ} \mathrm{C}$ and $40^{\circ} \mathrm{C}$ inlet temperature cases, but increases significantly with the $100^{\circ} \mathrm{C}$, evaporative case. The average Nusselt number increases nearly linearly with flow rate for the purely heating cases. For the evaporative case, this trend is different; the average Nusselt number is linear versus inlet flow rate for the low rotational 


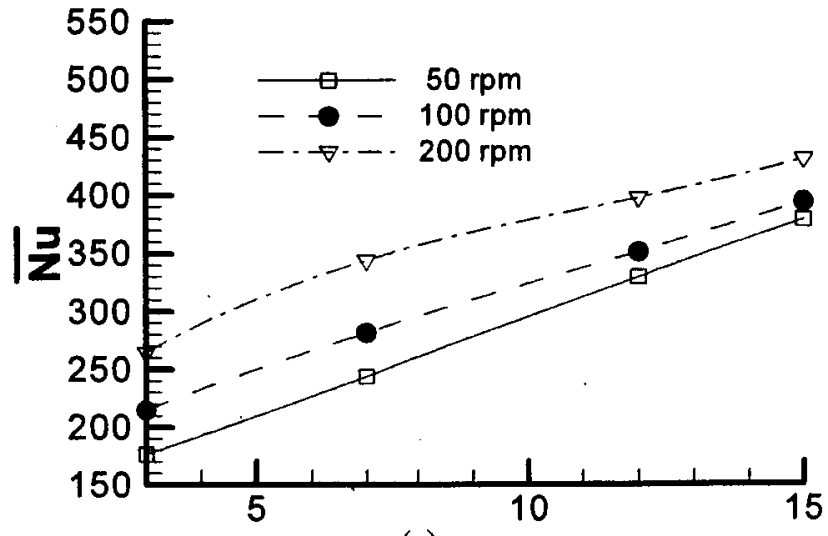

(a)

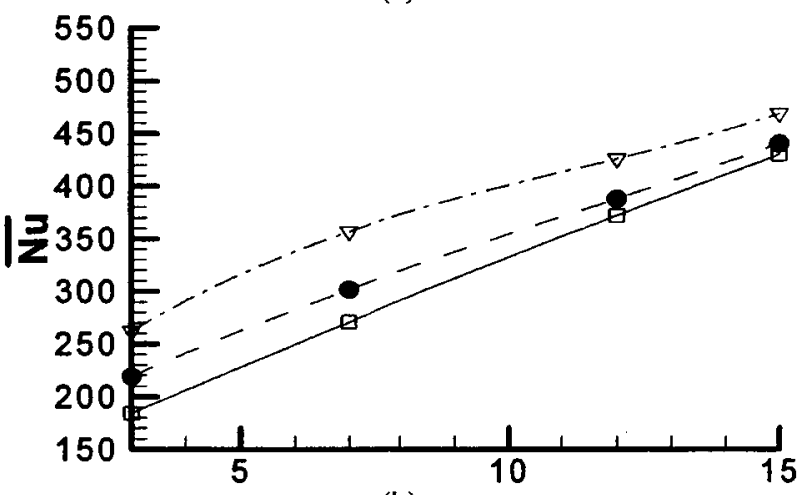

(b)

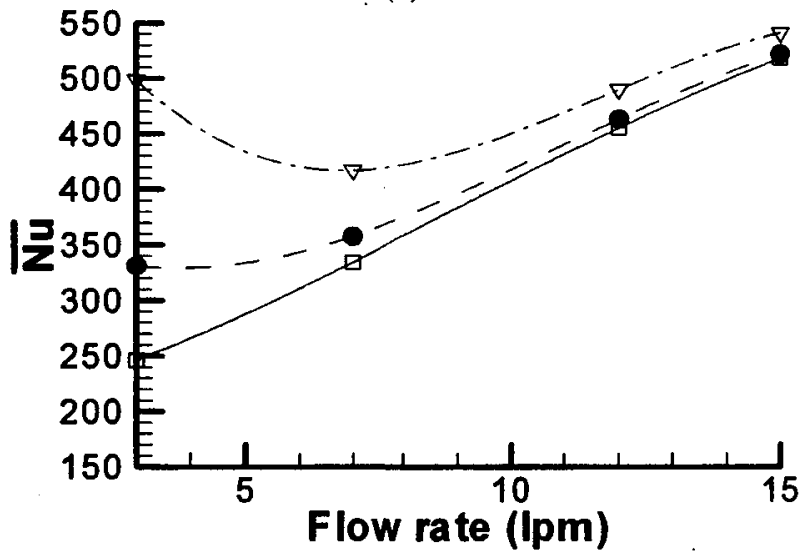

(c)

Figure 8: Average Nusselt number vs. flow rate at various inlet temperatures, $\delta_{\text {in }}=0.254 \mathrm{~mm}$, and a heating power of 4500 watts (a) $20^{\circ} \mathrm{C}$ (b) $40^{\circ} \mathrm{C}$ (c) $100^{\circ} \mathrm{C}$

speed cases and when the rotational speed is increased, a minimum overall heat transfer rate is observed at a slightly higher flow rate. The heat transfer is enhanced the most due to evaporative cooling at the lowest flow rates because of the nearly fully developed thermal field that exists.

The effects of the initial film thickness on the liquid film hydro and thermo characteristics are compared in Figure 9. The gap height used for all of the previous simulations was doubled and halved. The liquid film thickness behaves differently for the different gap heights in the inner inertia dominated region, and then the liquid film starts to behave similarly in the centrifugal dominated region. At the inner

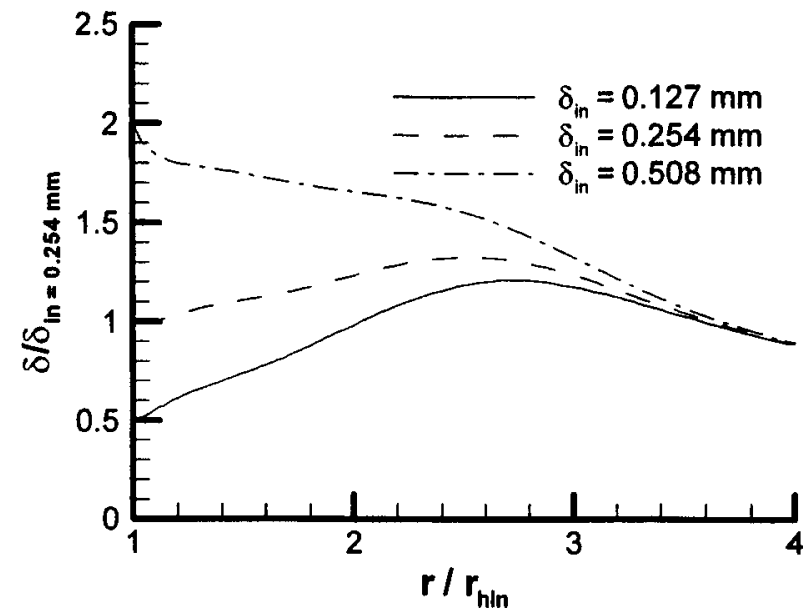

(a)

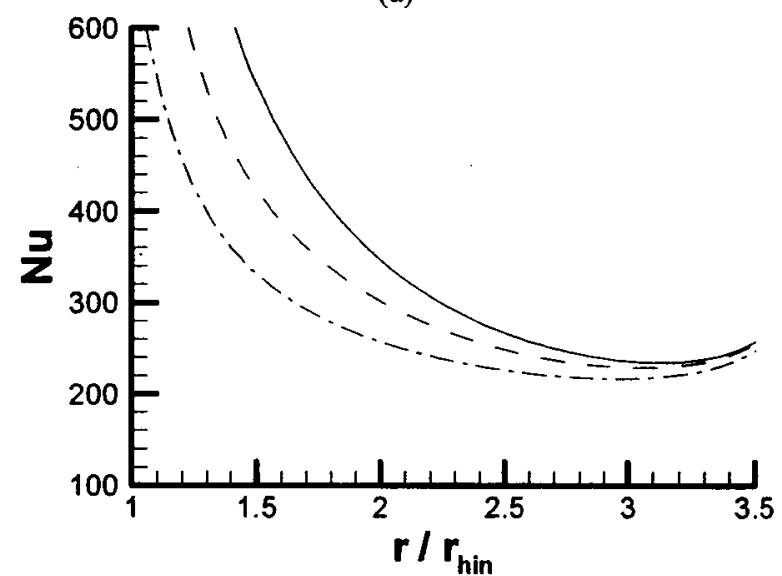

(b)

Figure 9: Effects of initial film thickness on (a) film thickness (b) local Nusselt number vs. radial location, for $7 \mathrm{lpm}, 100 \mathrm{rpm}$, at $40^{\circ} \mathrm{C}$ inlet temperature case and 4500 watts.

radii, near the liquid impingement, the film thickness initially increases with the tighter gap height and decreases with the looser gap height. This effect is because the fluid velocity is higher for the tighter gap height, and also the characteristic length for the viscous diffusion is less, therefore making the frictional losses much greater, which decelerates the flow much more rapidly than the greater gap height. Once the initial inertia effects are lost due to friction and the centrifugal effects are gained, due to rotation, the film characteristics become independent of the initial thickness of the liquid and just a function of the flow rate and the rotational speed. The local Nusselt number shows a very similar trend to the initial film thickness. Initially, the local Nusselt number is greater for the tighter collar gap height, because the film velocity is greater. The opposite effect is seen for a looser gap height. The local Nusselt numbers tend to the same line at greater radii because the liquid film characteristics become very similar in this region.

The conjugate heating effect is quantified in Figure 10. The same cases were run with heating applied at the disk surface and at the bottom of the disk. The Nusselt numbers are within 10 percent with and without the conjugate effect for 


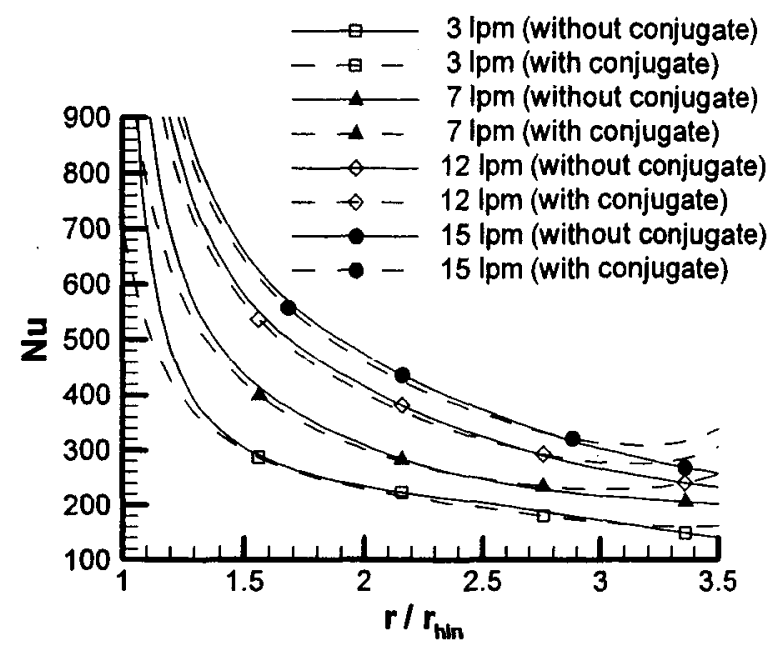

(a)

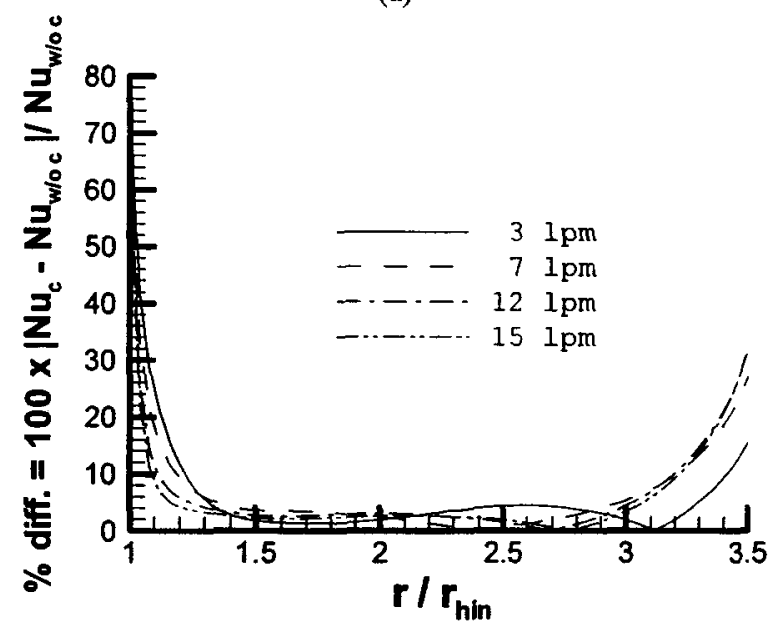

(b)

Figure 10: Conjugate effect on (a) local Nusselt number and (b) percent difference of local Nusselt Number vs. radial location for $\delta_{\mathrm{m}}=0.254 \mathrm{~mm}$, and $40^{\circ} \mathrm{C}$ inlet, 4500 watts at $100 \mathrm{rpm}$ for various flow rates.

about 80 percent of the disk. The conjugate effects are the greatest at the inner and outer radii, because of radial conduction through the disk. It is important to note that the average Nusselt numbers presented in this paper include the conjugate effect, and will be altered if the conjugate effect is not included. Also when the edge effects are modeled, the Nusselt number is within two percent of the model without the edge effects being modeled. The maximum difference in Nusselt number is at the outer edge of the heater because of the increased conjugate effect due to the convection heat transfer at the edge of the disk.

Doubling and halving the disk thickness examines the conjugate effect further. The effect of varying the disk thickness on Nusselt number is presented in Figure 11. The thicker the disk is, the more of an impact the conjugate effects has because of the increased cross-sectional area that allows radial conduction away from the heater. The Nusselt number is lower with increased disk thickness at the inner radii and higher at the outer radii. This happens because the fluid is being heated before the heater with conjugate heating, which

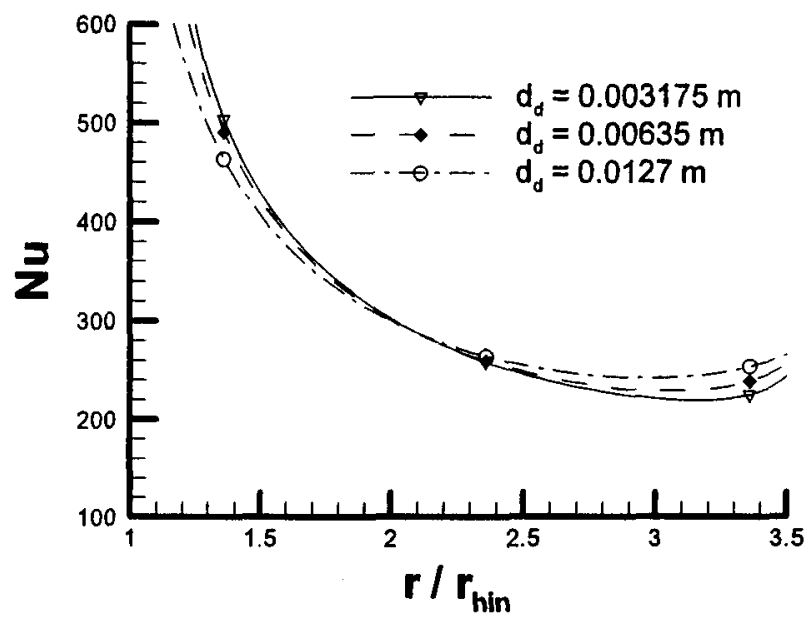

Figure 11: Conjugate effect on Nusselt number vs. radial location for various disk thickness at $7 \mathrm{lpm}, 100 \mathrm{rpm}, \delta_{\text {in }}=0.254 \mathrm{~mm}$, and $40^{\circ} \mathrm{C}$ inlet, and 4500 watts.

increases the film temperature, and heated less at the end of the heater because heating power is lost at the outer radii of the disk.

The effect of surface tension was also studied in these numerical simulations, which could not be done with experiments, as there is no control of such physical parameters with any given fluid. The film thickness and local Nusselt numbers varied by less than 0.1 percent for all of the cases when the surface tension was reduced by half or doubled. The results are not presented because of redundancy.

\subsection{Laminar to Turbulent transition}

Since the flow field was considered to be in a laminar regime for all of the present cases, a discussion of this assumption is needed. Azuma and Hoshino [16] and Azuma [17] studied the laminar to turbulent transition characteristics on a stationary and rotating disk, respectively, of an impinging jet. In these studies the inlet Reynolds number, the nozzle height and radius were varied to examine the effects on the laminar to turbulent transition. These studies looked at the surface waves and made conclusions relating the classification of surface waves to the turbulence intensities. The first class of waves are ' $D$ ' waves, which form concentric circles on the disk, then the increased disturbance of the fluid changes these waves into lattice-shaped (LS) waves. Finally, these waves are disturbed even further and become sandpaper-like (SL) waves. The transition from a laminar film to a turbulent film is characterized when the LS waves turn into SL waves, and the SL waves cover more than 50 percent of the disk in the circumferential direction. Azuma and Hoshino [16] found in the stationary cases, a critical discharge Reynolds number, $\mathrm{Re}^{*}$, of $7.4 \times 10^{4}$. The discharge Reynolds number takes into account a discharge coefficient, $c$, which is a function of flow rate, density, nozzle diameter and nozzle gap height from the disk. The critical discharge Reynolds number only changed slightly with the rotational rates presently investigated and is why the stationary cases were used for comparison [17]. This Reynolds number is different from the Reynolds number, Re, defined by Ozar et al. [15], which incorporates the mass flow rate and inlet radius of 
Table 1: Reynolds numbers, and laminar-turbulent transition radius from Azuma and Hoshino's (1984) analysis for the conditions considered in the present analysis.

\begin{tabular}{|c|c|c|c|c|c|c|}
\hline $\begin{array}{c}\text { Temp. } \\
\text { (Celsius) }\end{array}$ & $\begin{array}{c}\dot{V} \\
(\mathrm{pm})\end{array}$ & $\mathbf{c}$ & $\mathbf{R} \theta$ & $\mathbf{R}^{*}$ & $\mathbf{r}_{\mathrm{t}}^{*}$ & $\mathbf{r}_{\mathrm{t}} / \mathbf{r}_{\mathrm{hn}}$ \\
\hline 20 & 3 & 0.61 & 156 & $1.3 \mathrm{E}+04$ & Laminar & Laminar \\
\hline 20 & 7 & 0.61 & 364 & $2.9 \mathrm{E}+04$ & Laminar & Laminar \\
\hline 20 & 12 & 0.61 & 624 & $5.0 \mathrm{E}+04$ & Laminar & Laminar \\
\hline 20 & 15 & 0.61 & 779 & $6.3 \mathrm{E}+04$ & Laminar & Laminar \\
\hline 40 & 3 & 0.61 & 246 & $2.0 \mathrm{E}+04$ & Laminar & Laminar \\
\hline 40 & 7 & 0.61 & 574 & $4.6 \mathrm{E}+04$ & Laminar & Laminar \\
\hline 40 & 12 & 0.61 & 984 & $7.9 \mathrm{E}+04$ & 0.59 & 1.98 \\
\hline 40 & 15 & 0.61 & 1230 & $9.9 \mathrm{E}+04$ & 0.50 & 1.81 \\
\hline 100 & 3 & 0.61 & 548 & $4.4 \mathrm{E}+04$ & Laminar & Laminar \\
\hline 100 & 7 & 0.61 & 1278 & $1.0 \mathrm{E}+05$ & 0.49 & 1.79 \\
\hline 100 & 12 & 0.61 & 2190 & $1.8 \mathrm{E}+05$ & 0.35 & 1.53 \\
\hline 100 & 15 & 0.61 & 2738 & $2.2 \mathrm{E}+05$ & 0.30 & 1.42 \\
\hline
\end{tabular}

the impinging jet.

The flow is considered laminar when the discharge Reynolds number is below the critical value. When the discharge Reynolds number is greater than the critical value, there is a radius of transition from laminar to turbulent flow at some radial location on the disk. There is a non-dimensional radius of transition, $r_{t}{ }^{*}$, which is a function of the discharge Reynolds number, and the gap height and diameter of the nozzle. The Reynolds number, the discharge coefficient and the radius of transition, when applicable, are presented in Table 1. From this table, turbulence is only expected to influence the results of the higher flow rates and higher inlet fluid temperatures. Azuma [17] illustrated that the rotation tended to suppress the turbulent intensity when rotation was small (similar to rotational speeds used is this study), therefore pushing the transitional radius outward, and increase the turbulent intensity at higher rotational speed (rotational speeds greater than used in this study). It is important to note that the experiments were performed on a smooth glass disk, while the present numerical simulations are run on as laminar flow on an aluminum disk. Although laminar to turbulence transition is not considered in this numerical analysis, it is an area of interest for future research.

\section{Conclusions}

The liquid film characteristics studied in the range of 3 to $15 \mathrm{lpm}$, with disk rotational speeds of 50 to $200 \mathrm{rpm}$, and inlet temperatures varying from $20^{\circ} \mathrm{C}$ to $100^{\circ} \mathrm{C}$ are presented. The inertia of the fluid, the frictional losses as the liquid film flows over the disk and the centrifugal forces due to the rotation of the disk were all found to affect the film thickness. Some conclusions can be made about the fluid mechanics:

- The film thickness was found to decrease with increasing rotational speed. The transition point from the frictional loss dominated flow to the centrifugal force dominated flow was pushed radially outward with increasing flow rate.
- The increased inlet fluid temperature was found to decrease the film thickness because the frictional losses were less due to the decreased viscosity.

- The effect of the inlet gap height was found to effect the film thickness trend in the inner frictional loss dominated region but the film characteristics became similar once the centrifugal forces dominated the flow.

The heat transfer results were directly affected by the liquid film characteristics. Therefore, the heat transfer was affected by inlet temperature, inlet flow rate and the rotational speed. The conclusions that can be made about the heat transfer characteristics are:

- The heat transfer was enhanced with both increased rotational speed and increased flow rate. The enhancement of the heat transfer for the higher flow rate under increased rotational speed was not significant at inner radial distances.

- The increased temperature was found to increase the heat transfer because of the decreased film thickness.

- Evaporative effects were found to enhance the heat transfer more greatly at the lower flow rates, because the thermal field in the liquid film had fully developed characteristics closer the collar, therefore increasing the temperature gradient at the free surface, which enhances evaporative cooling.

- A reduced initial film thickness enhances the heat transfer at inner radial locations, but becomes less dependent on the inlet gap height radially outward on the disk, similar to the film characteristics.

- The conjugate effects made less than 10 percent difference in the local Nusselt in the middle of the heated section of the disk. However, the difference increased higher than 10 percent at the inner and outer 15 percent of the heated section of the disk.

From the above observations, when designing a system to utilize a controlled liquid impinging jet over a rotating surface, the point at which rotation starts to enhance the heat transfer varies with the inlet flow rate and rotational speed; this point is generally radially outward for the increase of these two parameters. From these observations, it is thought that for lower flow rates, smaller disks can be used, and still have the advantageous effects of rotation. On the other hand, for the higher flow rates and rotational speeds considered, a larger disk should be used to utilize the heat transfer enhancement due to rotation.

\section{Acknowledgment}

This work was sponsored by the NASA Microgravity Fluids Physics Program, Glenn Research Center, Cleveland Ohio, under the grant No. NCC3-789 with Dr. Subramanian Sankaran as the grant monitor.

\section{References}

[1] E.J. Watson, 1964, "The radial spread of a liquid jet over a horizontal plane," J. Fluid Mech., 20, pp. 481499. 
[2] Z. H. Chaudhury, 1964, "Heat transfer in a radial liquid jet," J. Fluid Mech., 20, pp. 501-511.

[3] A. D. D. Craik, R. C. Latham, M.J. Fawkes, and P.W.F. Gribbon, 1981, "The circular hydraulic jump," J. Fluid Mechanics, 112, pp. 347-362.

[4] A.Y. Tong, 2003, "On the impingement heat transfer of an oblique free surface plane jet," Int. J. Heat and Mass Transfer, 46, pp. 2077-2085.

[5] M.M. Rahman, A. Faghri, W.L. Hankey, and T.D. Swanson, 1990, "Computation of free surface flow of a liquid film at zero and normal gravity," Num. Heat Transfer, 17a, pp. 53-71.

[6] A. Faghri, S. Thomas, M.M. Rahman, 1993, "Conjugate heat transfer from a heated disk to a thin liquid film formed by a controlled impinging jet," J. Heat Transfer, 115, pp. 166-123.

[7] S. Thomas, A. Faghri, W. Hankey, 1991, "Experimental analysis and flow visualization of a thin liquid film on a stationary and rotating disk," Journal of Fluids Engineering, 133, pp. 73-80.

[8] S. Thomas, W. Hankey, A. Faghri, T. Swanson, 1990, "One-dimensional analysis of the hydrodynamic and thermal characteristics of thin film flows including the hydraulic jump and rotation," J. Heat Transfer, 112, pp. 728-735.

[9] M.M. Rahman, and A. Faghri, 1992, "Numerical simulation of fluid flow and heat transfer in a thin liquid film over a rotating disk," Int. J. Heat and Mass Transfer, 35, pp. 1441-1453.

[10] B. Ozar, B.M. Cetegen, A. Faghri, 2004, "Experiments on heat transfer in a thin liquid film flowing over a rotating disk," J. Heat Transfer, 126, pp. 184-192.

[11] C.W. Hirt and B.D. Nichols, 1981, "Volume of fluid (VOF) method for the dynamics of free boundaries," J. Comput. Phys., 39, pp. 201-225.

[12] D.L. Youngs, 1982, "Time-dependent multimaterial flow with large fluid distortion," Numerical Methods for Fluid Dynamics, K.W. Morton and M.J. Baines, ed. Academic Press.

[13] J.U. Brackbill, D.B. Kothe and C. Zemach, 1992, "A continuum method for modeling surface tension", J. Comput. Phys., 100, pp. 335-354.

[14] S.V. Patankar, 1980, Numerical Heat Transfer and Fluid Flow, Hemisphere, Washington, D.C.

[15] B. Ozar, B.M. Cetegen, A. Faghri, 2003, "Experiments on the flow of a thin liquid film over a horizontal stationary and rotating disk surface," Exp. in Fluids, 34, pp. 556-565.

[16] T. Azuma and T. Hoshino, 1984, "The radial flow of a thin liquid film, $1^{\text {st }}-4^{\text {th }}$ Reports," Bull JSME, 27, pp. 2739-2151.

[17] T. Azuma, 1989, "The transition from laminar to turbulent flow in the thin liquid film on a rotating disk," Seventh Symposium on Turbulent Shear Flows, Stanford University, Aug 21-23. 